proteasome inhibitor, was recently approved in the US and Canada for use in combination with lenalidomide and dexamethasone in patients with multiple myeloma who have received at least 1 prior therapy. The Keyhole limpet hemocyanin (KLH) model of $\mathrm{T}$ cell-dependent antigen response was used to determine if ixazomib depletes plasma cells resulting in a reduction of antibodies.

Methods Briefly, rats were immunised with KLH and TiterMax adjuvant then treated with ixazomib twice weekly until study termination.

Results Treatment with ixazomib significantly inhibited anti$\mathrm{KLH}$ antibodies by $34 \%(\mathrm{p}<0.05)$ versus vehicle. Additionally, $\mathrm{KLH}$ plasma cells quantified by ELISpot were decreased $78 \%$ $(\mathrm{p}<0.01)$ in the spleen and 53\% $(\mathrm{p}<0.01)$ in the bone marrow compared to control. To gain some understanding of the selectivity of plasma cell depletion total White Blood Cells, Red Blood Cells (RBC) Platelets, Neutrophils, and total Lymphocytes were quantified with small a reduction only seen in RBCs and platelets.

Conclusions Ixazomib depleted plasma cells resulting in reduced antibodies suggesting further preclinical studies are warranted in diseases with pathogenic antibodies such as SLE, RA, SS and solid organ transplant rejection.

\section{ABSENCE OF HOST CD137 SIGNALLING CONVERTS CHRONIC GVHD TOWARD ACUTE GVHD}

J Kim*, UJ Moon, H Kim, HR Cho, B Kwon. Ulsan University Hospital, Biomedical Research Centre, Ulsan, Republic of Korea

10.1136/lupus-2017-000215.49

Background and aims CD137 functions mainly as a costimulatory molecule for $\mathrm{T}$ cell activation. However, its functions have been found in a variety of other immune and nonimmune cells. Transfer of BM12 $\mathrm{CD}^{+}{ }^{+} \mathrm{T}$ cells into unirradiated, MHC II-mismateched C57BL/6 mice induces lupus-like chronic GVHD, which occurs because donor CD4 $+\mathrm{T}$ cells break host $\mathrm{B}$ cell tolerance with help from host $\mathrm{CD} 4^{+} \mathrm{T}$ cells.

Methods cGVHD was induced by transferring $8 \times 10^{7}$ cells/ mouse BM12 spleen/lymph node cells into C57BL/6 and CD137-/- mice. Serum samples were collected 2 wk after disease induction and assayed by ELISA for IgG1 anti-DNA autoantibody. Mouse body weight measured two times a week. Splenocytes were harvested 10 days after disease induction. After counting the number of total spleen and analysed by flow cytometry. Pathological scores of colons and livers. Colons and livers were harvested 10 days after disease induction.

Results We found that chronic GVHD was inhibited when CD $137^{-/-}$mice were used as the host in this chronic GVHD model. Instead, they exhibited evident loss of body weight, indicating that they had acute GVHD. Indeed, their splenocytes were markedly depleted and they had severe intestinal and liver GVHD. Consistent with these phenotype changes, there were increased numbers of Th1 and Th17 cells but decreased numbers of Treg cells in the spleen of CD $137^{-/}$ recipient mice 10 days after disease induction.

Conclusions Our results indicate that host CD137 signalling is a key factor to determine the fate of donor $\mathrm{CD}^{+} \mathrm{T}$ cells during GVHD course.

\section{EXPRESSION OF LY6C/6G DEFINES A NOVEL AIRE- DEPENDENT SUBSET OF MEDULLARY THYMIC EPITHELIAL CELLS WITH TOLEROGENIC FUNCTION}

J Morimoto, Y Nishikawa, H Nishijima, M Matsumoto*. Division of Molecular Immunology, Institute for Enzyme Research, Tokushima University

\subsection{6/lupus-2017-000215.50}

Medullary thymic epithelial cells (mTECs) are a heterogeneous population in terms of the spectrum of tissue-restricted Ags (TRAs) expressed from each cell for ensuring the elimination of autoreactive T-cells. Additionally, mTECs comprise cells at different developmental stages and/or in various activation conditions. Because of these heterogeneities, it is unclear whether mTECs are composed of any particular subsets possessing unique properties for their developmental pathway and/or immunological function. Here, we report a distinct mTEC subset characterised by expression of Ly6 family protein prior to and concomitant with Aire expression during its differentiation. Ly6C/6G+ mTECs, constituting 5\%-15\% of mature mTECs, were preferentially localised at the corticomedullary junction, and expressed high levels of TRAs and thymocyte-attracting chemokines. Remarkably, Ly6C/6G+ mTECs were absent in Aire-deficient mice, suggesting that this subset requires Aire and/or Aire+ mTECs for its production. Uniquely, Ly6C/6G + mTECs lack a post-Aire stage because of a tendency to die after Aire had been expressed. With a TCR-transgenic model in mice, we found that in vivo depletion of Ly6C/6G+ mTECs frequently induced organ-specific autoimmunity. We suggest that Ly6C/6G+ mTECs serve as an important source of TRAs for efficient cross-presentation during establishment of self-tolerance.

\section{INCREASED APOPTOSIS AND ABERRANT APOPTOSIS SIGNALLING PATHWAYS OF NATURAL CD4+CD25 +FOXP3+ REGULATORY T CELLS IN PATIENTS WITH SYSTEMIC LUPUS ERYTHEMATOSUS}

${ }^{1} \mathrm{~L}$ Na*, ${ }^{1} \mathrm{~F}$ Hao. ${ }^{1}$ Southwest Hospital Third Military Medical University, Dermatology, Chongqing, China

\subsection{6/lupus-2017-000215.51}

Background and aims Systemic Lupus Erythematosus(SLE) is a prototype of autoimmune disease.Decreased cell numbers and suppressive defects of naturally occurring $\mathrm{CD} 4{ }^{+} \mathrm{CD} 25^{+} \mathrm{FOX}$ $\mathrm{P}_{3}{ }^{+}$regulatory $\mathrm{T}$ cells(Tregs) play an important role in the breakdown of SLE immune tolenrance. We have peviously observed significantly increased apoptosis of peripheral blood $\mathrm{CD} 4^{+} \mathrm{T}$ cells in SLE patients.Our objective here was to detect the apoptosis of Tregs in SLE patients to see if it could contribute to reduced suppressive activity of Tregs, and further elucidate the genes and signalling pathways which trigger the apoptosis in these cells.

Methods The cell number and apoptosis rates of Tregs was respectively evaluated in SLE patients and normal controls (NCs) by FACS.The suppressive activity of Tregs was measured by coculture with $\mathrm{CD} 4^{+} \mathrm{CD} 25^{-} \mathrm{CD} 127^{\mathrm{dim} /} \mathrm{T}$ cells. The relationship of abnormal Tregs apoptosis with clinical parameters was analysed by correlation analysis.Gene expression profiles of unstimulated Tregs from active SLE patients and NCs were generated by microarray analysis.Differential genes expression were verified by real time-PCR. 
Results Tregs from SLE patients showed a significantly reduced number,elevated apoptosis rates and impared suppressive capacity compared with NCs. The increased Tregs apoptosis was negatively correlated with the total number of Tregs and positively correlated with disease activities.Microarray profiles of Tregs from SLE subjects reveal a cellular response that could make the cells sensitive to apoptosis,partially due to the stress responses,DNA-damaging and cytokine stimulation.

Conclusions This global picture of pathway-specific expression signatures is a step further dissecting Treg cells defects in the pathogenesis of SLE, and may shed light on the newly therapeutic strategies towards the aberrant Tregs apoptosis and reconstruction of SLE immune homeostasis.

\section{THE PATHOGENIC RELEVANCE OF T FOLLICULAR HELPER CELLS-PLASMABLASTS AXIS IN PATIENTS WITH SYSTEMIC LUPUS ERYTHEMATOSUS}

S Nakayamada*, S Kubo, M Yoshikawa, Y Miyazaki, K Sakata, K Nakano, S Iwata, I Miyagawa, K Saito, Y Tanaka. University of Occupational and Environmental Health, First Department of Internal Medicine, Kitakyushu, Japan

\subsection{6/lupus-2017-000215.52}

Background and aims The aim of this study was to assess the peripheral immune cell phenotypes in a correlation with clinical findings in patients with systemic lupus erythematosus (SLE).

Methods Peripheral blood mononuclear cells were obtained from 143 SLE patients and 26 healthy donors (HD). Circulating $\mathrm{B}, \mathrm{T}$ and dendritic cells were defined based on flow cytometric analysis for human immune system termed "the Human Immunology Project" proposed by the National Institutes of Health (NIH) and the Federation of Clinical Immunology Societies (FOCIS).

Results The proportions of $\mathrm{CD}^{+}{ }^{+} \mathrm{CD} 4^{+} \mathrm{CXCR}^{+} \mathrm{ICOS}^{+} \mathrm{T}$ follicular helper (Tfh) cells, but not $\mathrm{CD}^{+} \mathrm{CD} 4^{+} \mathrm{CXCR} 3^{+} \mathrm{CCR} 6$ Th1 and $\mathrm{CD}^{+}{ }^{+} \mathrm{CD} 4^{+} \mathrm{CXCR} 3{ }^{-} \mathrm{CCR} 6{ }^{+}$Th17 cells, were higher in SLE than the HD. The proportions of $\mathrm{CD}_{1}{ }^{+}$ $\mathrm{CD}_{20}{ }^{+} \mathrm{IgD}^{+} \mathrm{CD} 27^{+}$central memory $\mathrm{B}$ cells and $\mathrm{CD} 19^{+} \mathrm{CD} 20^{+} \mathrm{IgD}^{-} \mathrm{CD} 27^{-}$effector $\mathrm{B}$ cells were higher in SLE. The largest difference relative to the $\mathrm{HD}$ was observed in the proportion of $\mathrm{CD} 19^{+} \mathrm{CD} 20^{-} \mathrm{CD} 27^{+} \mathrm{CD} 38^{+}$plasmablasts, which was higher in SLE and correlated with BILAG index. The proportion of Tfh cells correlated with serum IgG level, and the proportion of activated Tfh cells correlated with serum anti-Sm antibody level. Among helper T cell subsets (Th1, Th17, Treg and Tfh), Tfh cells only showed positive correlation with the proportion of plasmablast $(\mathrm{r}=0.24, \mathrm{p}=0.02)$.

Conclusions Peripheral immuno-phenotyping confirmed the importance of Tfh-plasmablasts axis in patients with SLE, i.e. activation of Tfh cells correlated with autoantibody production while plasmablast did with disease activity of SLE. Our findings supported the relevance of Tfh-plasmablasts axis as a potential therapeutic target for SLE.

\section{REPOSITORY CORTICOTROPIN INJECTION EXERTS DIRECT ACUTE EFFECTS ON HUMAN B CELL GENE EXPRESSION DISTINCT FROM THE ACTIONS OF GLUCOCORTICOIDS}

${ }^{1} \mathrm{~N}$ Olsen*, ${ }^{1} \mathrm{~A}$ Benko, ${ }^{1} \mathrm{C}$ McAloose, ${ }^{2} \mathrm{~T}$ Sunyer, ${ }^{2} \mathrm{P}$ Becker, ${ }^{1} \mathrm{~W}$ Kovacs. ${ }^{1}$ Penn State Hershey, Medicine, Hershey, USA; ${ }^{2}$ Mallinckrodt Pharmaceuticals, Clinical Translational Research, Hazelwood Missouri, USA

\subsection{6/lupus-2017-000215.53}

Background and aims Repository corticotropin injection (RCI; H.P. Acthar Gel) is a porcine pituitary-derived ACTH preparation approved by the FDA for therapy in selected cases of SLE. Previous studies have shown that RCI directly inhibits human B cell function in vitro.

Methods We used RNA-Seq to identify elements of the transcriptome that are acutely modulated by RCI in human B cells activated in vitro by IL4 and CD40 ligand. We compared RCI effects to those of a synthetic glucocorticoid (dexamethasone; Dex) under the same conditions.

Results 115 unique gene transcripts were significantly and reproducibly upregulated by RCI after 24 hours in culture. Pathways analysis revealed that upregulated genes were overrepresented in "immune system response" $(2.8$-fold; $\mathrm{p}=0.026)$ and "response to stress" $(4.16$-fold; $p=0.0069) .74$ gene transcripts were down-regulated by RCI, and these were over-represented in two pathways: "immune system response" (2.91fold; $p=0.035)$ and "cellular process" (1.73-fold; $p=0.0036)$. In Dex-treated cells, 65 gene transcripts were upregulated and 23 gene transcripts were down-regulated. There was no overlap between the set of genes upregulated by RCI and Dex. Two genes (PARM1 and RANKL) were downregulated by both RCI and Dex. Pathways analysis of Dex- treated samples did not reveal significant overrepresentation of regulated genes in any specific ontologic pathway.

Conclusions These data suggest that RCI exerts direct effects on human B cells to acutely modulate gene expression. These effects are distinct from those of glucocorticoids, supporting potential differences in mechanism of action of these two agents for treatment of autoimmune diseases.

\section{$54 \quad$ R052 AUTOANTIBODIES ARISE FROM SELF-REACTIVE PROGENITORS IN A MOTHER OF A CHILD WITH NEONATAL LUPUS}

1J Reed*, ${ }^{2} \mathrm{M}$ Gorny, ${ }^{2} \mathrm{~L} \mathrm{Li},{ }^{3} \mathrm{~T}$ Cardozo, ${ }^{4} \mathrm{~J}$ Buyon, ${ }^{4} \mathrm{R}$ Clancy. ${ }^{1}$ Garvan Institute of Medical Research, Immunology, Darlinghurst, Australia; ${ }^{2}$ New York University School of Medicine, Pathology, New York, USA; ${ }^{3}$ New York University School of Medicine, Pharmacology, New York, USA; ${ }^{4}$ New York University School of Medicine, Medicine, New York, USA

\subsection{6/lupus-2017-000215.54}

Background and aims Autoantibodies targeting Ro52 occur in systemic lupus erythematosus, Sjogren's syndrome and idiopathic inflammatory myopathies. Yet the most compelling evidence for their pathogenesis is the development of cardiac conduction abnormalities, a manifestation of neonatal lupus, in 